\title{
Volume Holographic Optical Elements
}

\author{
Ching-Cherng Sun \\ National Central University \\ Institute of Optical Sciences \\ Chung-Li, 320 Taiwan \\ E-mail: ccsun@ios.ncu.edu.tw \\ Partha P. Banerjee \\ University of Dayton \\ Department of Electrical and Computer \\ Engineering \\ Dayton, Ohio 45469-0226 \\ E-mail: partha.banerjee@ notes.udayton.edu
}

Optical engineering continues to impact more and more modern devices for communication, color display, data storage, illumination, and remote sensing in the new millenium. The amalgamation of modern optics and associated microelectronic technologies has paved the road to greatly enhance new optoelectronic devices with practical applications. Modern optical technologies based on volume holography, both static and dynamic, have been developed over the past decade. Applications including high-density data storage, interconnections, spatial and temporal filters, beam shapers and combiners, gyros, and three-dimensional displays have been proposed and demonstrated. Simultaneously, more sensitive, efficient, and durable holographic materials have been developed. Novel optical elements based on volume holography perform unique functions, which have attracted much attention in modern science and engineering. Owing to the fast growth of the development of volume holographic optical elements, we dedicate this special section to expose researchers to the state of the art and to exchange ideas.

There are fourteen papers published in this special section. The subjects of the published papers cover material investigation, theoretical development, novel concepts, and applications based on holographic schemes. The first two papers present various state-of-the-art volume holographic elements. The first paper by Sinha et al. presents an overview of novel imaging systems that incorporate a volume hologram as one of the optical elements. A threedimensional volume holographic imaging element is proposed and analyzed. The second paper by Escuti et al. presents holographic fabrication of photonic crystals by providing a unified framework of design principles for the creation of two- and three-dimensional optical lattices, with an emphasis on how all five two-dimensional and all fourteen three-dimensional Bravais lattices may be formed with three and four coherent laser beams, respec- tively. Besides, they offer a comprehensive literature review and set forth the most pressing research issues in holographic photonic crystals.

The next two papers are concerned with the study of volume holographic recording materials. Huang et al. study photorefractive response under the influence of temperature in conjugation with reduction. In contrast, a study of static volume holographic materials is presented by Hsiao et al., who analyze the physical mechanism of holographic recording in phenanthrenequinone doped poly(methyl methacrylate) hybrid materials.

In the following five papers, various volume holographic optical elements performing wavelength filtering are reported. Yang et al. present an analysis of volume holographic long period gratings in photonic nanostructured fibers and waveguides. The photonic nanostructure could substantially enhance the tuning capability of the spectral response of the grating. Cao et al. propose a spectral device based on angular and wavelength multiplexing in volume holograms. The imaging function of the device is realized with convergent recording beams. The spectral system becomes compact without the focusing lens in general approaches. Havermeyer et al. propose and demonstrate a novel tunable optical filter, realized by angle tuning a volume holographic grating. The demonstrated 1510- to 1590 -nm tuning covers entire EDFA-C band, with small bandwidth variation and low insertion loss. Do et al. propose a Gaussian apodization technique for a volume holographic demultiplexer in the application of fiber communication. A 22-channel demultiplexer based on that grating is optically demonstrated. Chen et al. propose a design of a 2048-channel dense wavelength-division multiplexer with a channel spacing of $0.05 \mathrm{~nm}$ using reflection holograms in $\mathrm{LiNbO}_{3}$. The novel design is based on the calculation of Bragg selectivity of a reflection hologram with a VOHIL model. 
The next three papers are about spatial interconnection with the use of volume holographic optical elements. Liu et al. propose and demonstrate a scheme of image interconnections that transfers two-dimensional images between two ports by using a photorefractive $\mathrm{LiNbO}_{3}$ crystal and a $\mathrm{BaTiO}_{3}$ crystal. Han et al. propose and characterize remote multiplexing of holograms with random pattern reference from multimode fiber bundles. Angle, shift, and wavelength multiplexings at the remote input facet of the fiber bundle were examined and optimized for each case. The novel remote multiplexing method may provide a compact and integrated optic system for practical holographic interconnection and optical storage. Wang et al. propose a scheme for optical security with the interconnection between the randomly selected binary phase code and the signal. The decryption probability of the volume-holographic security device is less than $10^{-10}$ when the phase code is contained in only five rows.

The final two papers are concerned with the analysis of novel holograms. Banerjee et al. investigate holographic recording and reconstruction for edge-lit holograms recorded in a 90-degree geometry. Various cases of recording and readout that incorporate propagational diffraction have been modeled. It is shown that the 90-degree geometry can result in beam shaping, as evidenced through preliminary experimental results with photorefractive lithium niobate. Nguyen et al. propose a new approach for designing computer-generated holograms. An artificial neural network is used to initiate the genetic algorithm so that the high computation cost of genetic algorithms for synthesizing holograms is significantly reduced while the high diffraction efficiency and uniformity are obtained.

It is hoped that the collection of the fourteen papers in this special section will help readers in understanding the recent development of volume holographic optical elements and stimulate new research in the area. We hope that future work will lead to more exciting fundamental and applied discoveries in the area to stimulate future special sections on the topic. We hope that volume holo- graphic optical elements can find new and innovative applications in the areas of nano-optics and bio-optics.

Finally, we would like to thank all of the authors for their contributions and reviewers for their valuable comments and suggestions. We also thank Prof. O'Shea, managing editor Ms. Labes, and the Optical Engineering staff for their support for publishing this special section.

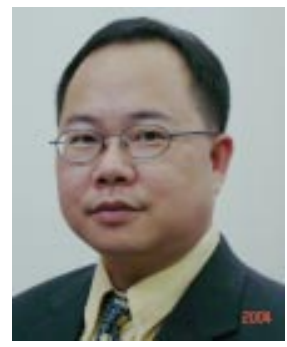

Ching-Cherng Sun received his BS in electrophysics from National Chiao Tung University in 1988 and his $\mathrm{PhD}$ in optical sciences from National Central University in 1993. In 1996, he joined the faculty of National Central University, Taiwan, and served as an associate professor at the Institute of Optical Sciences. He has been a full professor since 2002. He obtained the teaching award of the science college in 2001 . He is currently a member of the Optical Engineering Society of the Republic of China, SPIE, and OSA. He served as the secretary, the vice chairman, and the chairman of the SPIE Taiwan Chapter, respectively, from 1998 to 2003. His research interests are volume holographic optical elements, photorefractive devices, holography, LED lighting optics, optical information processing, optical metrology, micro-optics, fiber optics, and optical engineering.

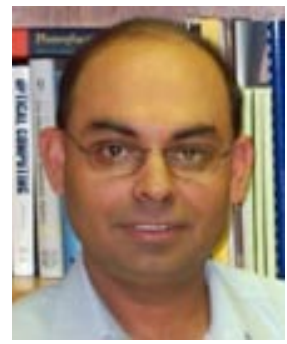

Partha P. Banerjee is professor and chair of the Department of Electrical and Computer Engineering at the University of Dayton. He received the BTech degree in electronics and telecommunication engineering from Indian Institute of Technology, Kharagpur, India, in 1979. He received the MS and $\mathrm{PhD}$ degrees in electrical and computer engineering from the University of lowa in 1980 and 1983, respectively. He served as a faculty member at the University of Alabama in Huntsville from 1991 to 2000, and at Syracuse University from 1984 to 1991. His area of research interest is nonlinear optics and photorefractives. He was the recipient of the National Science Foundation Presidential Young Investigator Award in 1987. He is a fellow of the Optical Society of America and of SPIE, and is a senior member of IEEE. To date he has more than 90 refereed journal publications and has authored four textbooks. 